\title{
RIEMANNIAN SUBMERSION AND THE LAPLACE-BELTRAMI OPERATOR
}

\author{
By Yosio Mutō
}

\section{Introduction.}

In the present paper we consider only Riemannian submersions $\pi:(\tilde{M}, \tilde{g}) \rightarrow$ $\left(B,{ }^{B} g\right)$ such that fibers $F$ are complete and connected and imbedded in $(\tilde{M}, \tilde{g})$ regularly as totally geodesic submanifolds.

It is well-known that, if $\varphi$ is an eigenfunction of the Laplacian in $\left(B,{ }^{B} g\right)$, the lift $\tilde{\varphi}=\varphi^{L}$ is also an eigenfunction of the Laplacian in $(\tilde{M}, \tilde{g})$ with the same eigenvalue [1]. The purpose of the present paper is to find corresponding relations in the case of $p$-forms. For $p$-forms we get a little more complicated result. If a $p$-form $\omega$ is an eigenelement of the Laplace-Beltrami operator $\Delta$ in $\left(B,{ }^{B} g\right)$, the horizontal lift $\widetilde{\omega}=\omega^{L}$ is not always an eigenelement of the Laplace-Beltrami operator $\tilde{\Delta}$ in $(\tilde{M}, \tilde{g})$. In order that $\tilde{\omega}$ be an eigenelement with the same eigenvalue as $\omega, \omega$ must satisfy a necessary and sufficient condition which is obtained in $\S 4$ of the present paper.

In $\S 1$ we recall some properties of Riemannian submersions with totally geodesic fibers. There we use local coordinates adapted to the Riemannian submersion. In $\S 2$ fundamental formulas in covariant differentiation are given. In $\S 3$ a relation between $\tilde{\Delta} \widetilde{\omega}$ and $\Delta \omega$ is obtained when $\widetilde{\omega}=\omega^{L}$. In $\S 4$ a necessary and sufficient condition to be satisfied by $\omega$ such that $\Delta \omega=\lambda \omega$ is obtained in order that $\tilde{\omega}=\omega^{L}$ satisfy $\tilde{\Delta} \tilde{\omega}=\lambda \tilde{\omega}$. A simple sufficient condition is also obtained. As an application harmonic forms are studied in some special case.

Remark. In the present paper lift always means horizontal lift.

\section{$\S 1$. Riemannian submersions with totally geodesic fibers.}

Riemannian submersions were studied extensively by the authors R. H. Escobales [2], S. Ishihara [3], S. Ishihara and M. Konishi [4], Y. Mutō [5], T. Nagano [7], B. O'Neill [8], K. Yano and S. Ishihara [10], [11] and others.

Riemannian submersions considered in the present paper are limited to those with totally geodesic fibers only, and this means that the tensor $T$ of B. O'Neill vanishes [8]. Tensors in the total manifold $\tilde{M}$, in the base manifold $B$ or in a

Received May 21, 1977. 
fiber $F$ are written in such letters as $\tilde{S},{ }^{B} S$ or ${ }^{F} S$ respectively, but, if there is no possibility of confusion, tensors ${ }^{B} S$ in $B$ are written $S$ for short. The Riemannian metrics on $\tilde{M}, B$ and $F$ are denoted respectively by $\tilde{g},{ }^{B} g$ and ${ }^{F} g$.

Let $\widetilde{W}$ be any vector field on $\tilde{M}, \tilde{E}$ any horizontal vector field on $\tilde{M}$ and $\tilde{X}$ any vertical vector field on $\tilde{M}$. Then for example, from any $(1,1)$-tensor field $\tilde{S}$ on $\tilde{M}$, we get four $(1,1)$-tensor fields $\widetilde{S}_{H}{ }^{H}, \widetilde{S}_{H}{ }^{V}, \widetilde{S}_{V}{ }^{H}, \widetilde{S}_{V}{ }^{V}$ such that

$$
\begin{gathered}
\tilde{S}=\tilde{S}_{H}{ }^{H}+\widetilde{S}_{H}{ }^{V}+\widetilde{S}_{V}{ }^{H}+\widetilde{S}{ }^{V}, \\
\widetilde{S}_{H}{ }^{H} \tilde{X}=\widetilde{S}_{H}{ }^{V} \tilde{X}=\widetilde{S}_{V}{ }^{H} \tilde{E}=\widetilde{S}_{V}{ }^{V} \tilde{E}=0, \\
\tilde{g}\left(\widetilde{S}_{H}{ }^{H} \widetilde{W}, \tilde{X}\right)=0, \quad \tilde{g}\left(\tilde{S}_{V}{ }^{H} \widetilde{W}, \tilde{X}\right)=0, \\
\tilde{g}\left(\widetilde{S}_{H}{ }^{V} \widetilde{W}, \tilde{E}\right)=0, \quad \tilde{g}\left(\widetilde{S}_{V}{ }^{V} \widetilde{W}, \tilde{E}\right)=0 .
\end{gathered}
$$

It is easy to see that such a decomposition of $\tilde{S}$ is unique. Similarly, if $\tilde{S}$ is a $(0,2)$-tensor field, we have a unique decomposition

$$
\tilde{S}=\tilde{S}_{H H}+\tilde{S}_{H V}+\tilde{S}_{V H}+\tilde{S}_{V V} .
$$

The (0, 2)-tensor field and the (2,0)-tensor field associated with the Riemannian metric $\tilde{g}$ are decomposed into $\tilde{g}_{H H}+\tilde{g}_{V V}$ and $\tilde{g}^{H H}+\tilde{g}^{V V}$ respectively since $\tilde{g}_{H V}$ and $\tilde{g}^{H V}$ vanish.

We define a tensor field $\widetilde{R}$ with the following property.

$\tilde{R}$ has only one non-vanishing part, namely,

$$
\widetilde{R}=\widetilde{R}_{H H^{V}} \text {. }
$$

Let $\tilde{A}$ be the tensor field $A$ in O'Neill's paper [8]. Let $\tilde{E}, \tilde{F}$ be any horizontal vector fields and $\tilde{X}$ any vertical vector field. Then $\tilde{R}$ satisfies

$$
\tilde{A}_{\widetilde{E}} \tilde{F}=-\frac{1}{2} \widetilde{R}_{\widetilde{E}} \widetilde{F}, \quad \tilde{g}\left(\tilde{A}_{\widetilde{E}} \tilde{X}, \tilde{F}\right)=\frac{1}{2} \tilde{g}\left(\widetilde{R}_{\widetilde{E}} \widetilde{F}, \tilde{X}\right) .
$$

We assume that $\tilde{M}$ is covered by a set $\{V\}$ of coordinate neighborhoods with the following property. $\pi V$ is a coordinate neighborhood of $B$ and for any point $P \in V$ we have local coordinates $P \Leftrightarrow\left(x^{1}, \cdots, x^{n}, y^{1}, \cdots, y^{m}\right)=\left(x^{1}, \cdots, x^{n}, x^{n+1}, \cdots\right.$, $\left.x^{n+m}\right)$ such that $\pi P \Leftrightarrow\left(x^{1}, \cdots, x^{n}\right)$. If we use the natural frame attached to such a coordinate neighborhood $V$, the components $\left(\tilde{X}^{1}, \cdots, \tilde{X}^{n}, \tilde{X}^{n+1}, \cdots, \tilde{X}^{n+m}\right)$ of a vertical vector $\tilde{X}$ satisfy $\tilde{X}^{h}=0$ where $h=1, \cdots, n$.

We use indices in the following ranges:

$$
\begin{aligned}
& h, \imath, j, \cdots, r, s, \imath, \cdots=1, \cdots, n, \\
& \kappa, \lambda, \mu, \cdots, \rho, \sigma, \tau, \cdots=n+1, \cdots, n+m, \\
& A, B, C, \cdots, R, S, T, \cdots=1, \cdots, n+m .
\end{aligned}
$$

Then the covariant components of the Riemannian metric $\tilde{g}$ are $\tilde{g}_{C B}$, or separ- 
ately, $\tilde{g}_{j i}, \tilde{g}_{j \lambda}, \tilde{g}_{\mu \imath}, \tilde{g}_{\mu \lambda}$ where $\tilde{g}_{j \lambda}=\tilde{g}_{\lambda j}$. The covariant components ${ }^{F} g_{\mu \lambda}$ of ${ }^{F} g$ satisfy ${ }^{F} g_{\mu \lambda}=\tilde{g}_{\mu \lambda \text {. }}$ The inverse matrix of $\left({ }^{F} g_{\ell \lambda \lambda}\right)$ is denoted $\left({ }^{F} g^{\mu \lambda}\right)$.

Now we define $\Gamma_{\imath}^{\kappa}$ by

$$
\Gamma_{\imath}^{\kappa}={ }^{F} g^{\kappa \tau} \tilde{g}_{i \tau}
$$

For any vector $\widetilde{W}$ we have $\widetilde{W}=\widetilde{W}^{H}+\widetilde{W}^{V}$. If $\widetilde{W}^{A}$, namely $\widetilde{W}^{h}$ and $\widetilde{W}^{n}$, are the components of $\widetilde{W}$, and the components of $\widetilde{W}^{H}$ and $\widetilde{W}^{V}$ are denoted $\left(\widetilde{W}^{H}\right)^{A}$ and $\left(\widetilde{W}^{V}\right)^{A}$ respectively, then we have

$$
\left(\widetilde{W}^{H}\right)^{h}=\widetilde{W}^{h}, \quad\left(\widetilde{W}^{H}\right)^{\kappa}=-\Gamma_{\imath}^{\kappa} \widetilde{W}^{i},
$$

$$
\left(\widetilde{W}^{V}\right)^{n}=0, \quad\left(\widetilde{W}^{v}\right)^{\kappa}=\widetilde{W}^{\kappa}+\Gamma_{\imath}^{\kappa} \widetilde{W}^{i} .
$$

For any covariant vector $\tilde{U}$ we have $\tilde{U}=\tilde{U}_{H}+\tilde{U}_{V}$ and

$$
\left(\tilde{U}_{H}\right)_{h}=\tilde{U}_{h}-\Gamma_{h}^{\kappa} \tilde{U}_{\kappa}, \quad\left(\tilde{U}_{H}\right)_{n}=0,
$$

$$
\left(\tilde{U}_{V}\right)_{h}=\Gamma_{h}^{\kappa} \tilde{U}_{\kappa}, \quad\left(\tilde{U}_{V}\right)_{\kappa}=\tilde{U}_{\kappa} .
$$

Using such local coordinates and natural frames we can deduce that $\tilde{R}$ has components

$$
\widetilde{R}_{j i}{ }^{\kappa}=\left(\widetilde{R}_{H H}{ }^{V}\right)_{j i}{ }^{\kappa}=D_{j} \Gamma_{\imath}^{\kappa}-D_{i} \Gamma_{j}^{\kappa}
$$

where

$$
D_{i}=\partial_{i}-\Gamma_{i}^{\lambda} \partial_{\lambda}, \quad \partial_{i}=\partial / \partial x^{2}, \quad \partial_{\lambda}=\partial / \partial x^{\lambda} .
$$

All other components of $\widetilde{R}$ vanish and we shall write $R_{j i}{ }^{\kappa}$ for the sake of convenience instead of $\widetilde{R}_{j i}{ }^{k}$.

For the Riemannian metric ${ }^{B} g$ on the base manifold $B$, we have

$$
{ }^{B} g_{j i}=\tilde{g}_{j i}-\Gamma_{j}^{\mu} \Gamma_{\imath}^{\lambda} \tilde{g}_{\mu \lambda}, \quad{ }^{B} g^{j i}=\tilde{g}^{j i} .
$$

It is easy to observe that ${ }^{B} g_{j i}=\left(\tilde{g}_{H H}\right)_{j i},{ }^{B} g^{j i}=\left(\tilde{g}^{H H}\right)^{j i}$. Moreover we have

$$
\begin{gathered}
{ }^{F} g_{\mu \lambda}=\left(\tilde{g}_{V V}\right)_{\mu \lambda}, \quad{ }^{F} g^{\mu \lambda}=\left(\tilde{g}^{V V}\right)^{\mu \lambda}=\tilde{g}^{\mu \lambda}-\Gamma_{t}^{\mu} \Gamma_{s}^{\lambda} \tilde{g}^{t s}, \\
\tilde{g}^{j \lambda}=-\Gamma_{t}^{\lambda} \tilde{g}^{j t} .
\end{gathered}
$$

As there is no possibility of confusion we shall write $g_{j i}, g^{j i}, g_{\mu \lambda}, g^{\mu \lambda}$ for ${ }^{B} g_{j i}$, ${ }^{B} g^{j i},{ }^{F} g_{\mu \lambda},{ }^{F} g^{\mu \lambda}$ respectively.

With the use of these components we can raise and lower indices of $\widetilde{R}_{j 2}{ }^{\kappa}$ and get tensor fields such as $\widetilde{R}_{H}{ }^{H V}, \widetilde{R}_{H H V}$ whose components are $R_{\jmath}{ }^{\imath \kappa}=R_{\jmath t}{ }^{\kappa} g^{t \imath}, R_{\jmath i \kappa}=$ $R_{j i}^{\tau} g_{\tau \kappa} . \quad \tilde{R}^{H H V}, \tilde{R}_{V}^{H H}$ are defined similarly. 


\section{§ 2. Fundamental formulas in covariant differentiation.}

Fundamental formulas of covariant differentiation have been obtained by B. O'Neill [8]. The following is only a translation into our terminology where $\widetilde{W}$ is a vector field and $\tilde{U}$ a 1 -form.

$$
\begin{aligned}
& \left((\tilde{\nabla} \widetilde{W})_{H}{ }^{H}\right)_{j}{ }^{h}=D_{j}\left(\widetilde{W}^{H}\right)^{h}+{ }^{B}\left\{\begin{array}{c}
h \\
j t
\end{array}\right\}\left(\widetilde{W}^{H}\right)^{t}+\frac{1}{2} R_{j}{ }^{h}\left(\widetilde{W}^{V}\right)^{\tau}, \\
& \left((\tilde{\nabla} \widetilde{W})_{H}{ }^{V}\right)_{\jmath}{ }^{\kappa}=D_{j}\left(\widetilde{W}^{V}\right)^{\kappa}+\partial_{\tau} \Gamma_{\jmath}^{\kappa}\left(\widetilde{W}^{V}\right)^{\tau}-\frac{1}{2} R_{j t}{ }^{\kappa}\left(\widetilde{W}^{H}\right)^{t}, \\
& \left((\tilde{\nabla} \widetilde{W})_{V}{ }^{H}\right)_{\mu}{ }^{h}=\partial_{\mu}\left(\widetilde{W}^{H}\right)^{h}+\frac{1}{2} R_{t}{ }^{h}{ }_{\mu}\left(\widetilde{W}^{H}\right)^{t}, \\
& \left((\tilde{\nabla} \widetilde{W})_{V}{ }^{V}\right)_{\mu^{2}}{ }^{\kappa}=\partial_{\mu}\left(\widetilde{W}^{V}\right)^{\kappa}+{ }^{F}\left\{\begin{array}{c}
\kappa \\
\mu \tau
\end{array}\right\}\left(\widetilde{W}^{V}\right)^{\tau}, \\
& \left((\tilde{V} \tilde{U})_{H H}\right)_{\jmath \imath}=D_{j}\left(\tilde{U}_{H}\right)_{i}-{ }^{B}\left\{\begin{array}{c}
t \\
\jmath
\end{array}\right\}\left(\tilde{U}_{H}\right)_{t}+\frac{1}{2} R_{j i}{ }^{\tau}\left(\tilde{U}_{V}\right)_{\tau}, \\
& \left((\tilde{\nabla} \tilde{U})_{H V}\right)_{\jmath \lambda}=D_{j}\left(\tilde{U}_{V}\right)_{\lambda}-\partial_{\lambda} \Gamma_{j}^{\tau}\left(\tilde{U}_{V}\right)_{\tau}-\frac{1}{2} R_{\jmath}{ }_{\lambda}\left(\tilde{U}_{H}\right)_{t}, \\
& \left((\tilde{\nabla} \tilde{U})_{V H}\right)_{\mu i}=\partial_{\mu}\left(\tilde{U}_{H}\right)_{i}-\frac{1}{2} R_{\imath}{ }^{t}{ }_{\mu}\left(\tilde{U}_{H}\right)_{t}, \\
& \left((\tilde{V} \tilde{U})_{V V}\right)_{\mu \lambda}=\partial_{\mu}\left(\tilde{U}_{V}\right)_{\lambda}-{ }^{F}\left\{\begin{array}{c}
\tau \\
\mu \lambda
\end{array}\right\}\left(\tilde{U}_{V}\right)_{\tau} .
\end{aligned}
$$

From these formulas we can get similar formulas of covariant differentiation of tensor fields.

From the tensor field $\tilde{R}$ we get two important tensor fields by covariant differentiation. They are $(\tilde{\nabla} \tilde{R})_{H H H}{ }^{V}$ and $(\tilde{V} \tilde{R})_{V H H}{ }^{V}$ whose leading components are

$$
R_{k j i}{ }^{k}=\left((\tilde{V} \tilde{R})_{H H H}{ }^{V}\right)_{k j i}{ }^{k}, \quad R_{\nu j i}{ }^{k}=\left((\tilde{V} \tilde{R})_{V H H}{ }^{V}\right)_{\nu j i}{ }^{k} \text {. }
$$

In view of (1.1) and the fundamental formulas given above we have

$$
\begin{aligned}
& R_{k j i}{ }^{\kappa}=D_{k} R_{j i}{ }^{\kappa}-{ }^{B}\left\{\begin{array}{c}
t \\
k j
\end{array}\right\} R_{t i}{ }^{\kappa}-{ }^{B}\left\{\begin{array}{c}
t \\
k i
\end{array}\right\} R_{j t}{ }^{\kappa}+\partial_{\tau} \Gamma_{k}{ }_{k} R_{j i}{ }^{\tau}, \\
& R_{\nu j i}{ }^{\kappa}=\partial_{\nu} R_{j i}{ }^{\kappa}+{ }^{F}\left\{\begin{array}{c}
\kappa \\
\nu \tau
\end{array}\right\} R_{j i}{ }^{\tau}+\frac{1}{2}\left(R_{j}{ }^{t} R_{i t}{ }^{\kappa}-R_{\imath}{ }_{\nu}{ }_{\nu} R_{j t}{ }^{\kappa}\right) .
\end{aligned}
$$

We can raise and lower indices of such tensor fields and define for example

$$
R_{k j}{ }^{2 \kappa}=R_{k j t}{ }^{\kappa} g^{t \imath}, \quad R_{\nu j i \kappa}=R_{\nu j i}{ }^{\tau} g_{\tau \kappa} .
$$


The following identities are obtained by direct computation or by applying identities satisfied by curvature tensors to formulas to be given a little later.

$$
\begin{gathered}
R_{k j i}{ }^{\kappa}+R_{j i k}{ }^{\kappa}+R_{i k j}{ }^{\kappa}=0, \\
R_{\mu j i \lambda}+R_{\lambda j i \mu}=0 .
\end{gathered}
$$

Relations between the curvature tensors $\tilde{K}_{D C B A},{ }^{B} K_{k j \imath h},{ }^{F} K_{\nu \mu \lambda \kappa}$ of $(\tilde{M}, \tilde{g})$, $\left(B,{ }^{B} g\right),\left(F,{ }^{F} g\right)$ have been obtained by B. O'Neill [8]. In our terminology they are

$$
\begin{aligned}
& \left(\tilde{K}_{H H H H}\right)_{k j i h}={ }^{B} K_{k j i h}-\frac{1}{4}\left(R_{j i}{ }^{\tau} R_{k h \tau}-R_{k \imath}{ }^{\tau} R_{j h \tau}\right)+\frac{1}{2} R_{k j}{ }^{\tau} R_{i h \tau}, \\
& \left(\tilde{K}_{H H H V}\right)_{k j i \kappa}=\frac{1}{2} R_{i k j \kappa}, \\
& \left(\tilde{K}_{H V H V}\right)_{k \mu \imath \kappa}=\frac{1}{2} R_{\mu k \imath \kappa}-\frac{1}{4} R_{k}{ }^{t}{ }_{\mu} R_{\imath t \kappa}, \\
& \left(\tilde{K}_{V V H V}\right)_{\nu \mu \imath \kappa}=0 \\
& \left(\tilde{K}_{V V V V}\right)_{\nu \mu \lambda \kappa}={ }^{F} K_{\nu \mu \lambda \kappa} .
\end{aligned}
$$

For the Ricci tensors $\widetilde{R \imath c}=\widetilde{K}_{H I I}+\tilde{K}_{H V}+\widetilde{K}_{V H}+\widetilde{K}_{V V},{ }^{B} R i c,{ }^{F} R \imath c$ of $(\tilde{M}, \tilde{g}),\left(B,{ }^{B} g\right)$, $\left(F,{ }^{F} g\right)$ we have

$$
\begin{aligned}
& \left(\tilde{K}_{H H}\right)_{j i}={ }^{B} K_{j i}-\frac{1}{2} R_{j}^{t \tau} R_{i t \tau}, \\
& \left(\tilde{K}_{H V}\right)_{j \lambda}=\frac{1}{2} g^{t s} R_{t \jmath s \lambda}, \\
& \left(\tilde{K}_{V H}\right)_{\mu i}=\frac{1}{2} g^{t s} R_{t \imath s \mu}, \\
& \left(\tilde{K}_{V V}\right)_{\mu \lambda \lambda}={ }^{F} K_{\mu \lambda}+\frac{1}{4} R^{t s}{ }_{\mu} R_{t s \lambda}
\end{aligned}
$$

where ${ }^{B} K_{j i},{ }^{F} K_{\mu \lambda}$ are the components of ${ }^{B} R i c,{ }^{F} R \imath c$ respectively.

From the above formulas we get

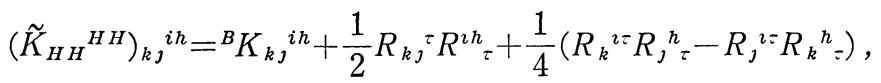

$$
\begin{aligned}
& \left(\tilde{K}_{H V}^{H H}\right)_{k \mu}{ }^{i h}=\frac{1}{2} R_{k}{ }^{i n}{ }^{\prime}, \\
& \left(\tilde{K}_{V V}{ }^{H H}\right)_{\nu \mu}{ }^{i h}=R_{\nu}{ }^{i h}{ }_{\mu}-\frac{1}{4}\left(R_{t}{ }^{2}{ }_{\nu} R^{t h}{ }_{\mu}-R_{t}{ }^{2}{ }_{\mu} R^{t h}{ }_{\nu}\right),
\end{aligned}
$$




$$
\begin{aligned}
& \left(\tilde{K}_{H I}{ }^{H}\right)_{\jmath}{ }^{\imath}={ }^{B} K_{\jmath}{ }^{2}-\frac{1}{2} R_{t \jmath}{ }^{\tau} R^{t \imath}{ }_{\tau}, \\
& \left(\tilde{K}_{V}{ }^{H}\right)_{\mu}{ }^{\imath}=\frac{1}{2} R_{t}{ }^{\imath t}{ }_{\mu}=-\frac{1}{2} R_{t}{ }^{t \imath}{ }^{\mu} .
\end{aligned}
$$

\section{§ 3. The Laplace-Beltrami operator in Riemannian submersion.}

In $\left(B,{ }^{B} g\right)$ let $\omega=U_{\imath_{1} \cdots \imath} d x^{\imath_{1}} \wedge \cdots \wedge d x^{\imath} p$ be a $p$-form and $\Delta$ the Laplace-Beltrami operator. Then we have

$$
\begin{aligned}
(\Delta U)_{i_{1} \cdots \imath} p & -\nabla_{t} \nabla^{t} U_{\imath_{1} \cdots \imath}+\sum_{a=1}^{p}{ }^{B} K_{\imath_{a}}{ }^{t} U_{\imath_{1} \cdots t \cdots \imath} p \\
& +\sum_{1 \leqq a<b \leqq p}{ }^{B} K_{\imath_{a}{ }^{\imath} b}{ }^{t s} U_{\imath_{1} \cdots t \cdots s \cdots \imath} .
\end{aligned}
$$

Similarly in $(\tilde{M}, \tilde{g})$ we have for $\tilde{\omega}=\tilde{U}_{A_{1} \cdots A_{p}} d x^{A_{1}} \wedge \cdots \wedge d x^{A p}$

$$
\begin{aligned}
(\tilde{\Delta} \tilde{U})_{A_{1} \cdots A_{p}}= & -\tilde{V}_{T} \tilde{V}^{T} \tilde{U}_{A_{1} \cdots A_{p}}+\sum_{a=1}^{p} \tilde{K}_{A_{a}}{ }^{T} \tilde{U}_{A_{1} \cdots T \cdots A_{p}} \\
& +\sum_{1 \leq a<b \leq p} \tilde{K}_{A_{a} A_{b}}{ }^{T S} \tilde{U}_{A_{1} \cdots T \cdots S \cdots A_{p}} .
\end{aligned}
$$

We now decompose $\tilde{\Delta} \tilde{U}$ into parts,

$$
\tilde{\Delta} \tilde{U}=(\tilde{\Delta} \tilde{U})_{H \cdots H H}+(\tilde{\Delta} \tilde{U})_{H \cdots H V}+\cdots+(\tilde{\Delta} \tilde{U})_{V \cdots V V} .
$$

But what we want to get is $\tilde{\Delta} \tilde{U}$ when $\tilde{U}$ is the lift, $\tilde{U}=U^{L}$. As $\tilde{U}$ satisfies $\tilde{U}=$ $\widetilde{U}_{H \cdots I I}$ in this case, namely, any part such as $\widetilde{U}_{H \cdots V \cdots H}$ vanishes, any part of $\tilde{\Delta} \tilde{U}$ where $V$ appears more than twice in the subscript vanishes in view of fundamental formulas of differentiation. Hence we need only to calculate $(\tilde{\Delta} \tilde{U})_{H \cdots H H H}$, $(\tilde{\Delta} \tilde{U})_{H \cdots H H V}$ and $(\tilde{\Delta} \tilde{U})_{H \cdots H V V}$.

In order to obtain for example $(\tilde{\Delta} \tilde{U})_{H \cdots H}$ we first calculate the second derivative $\tilde{\nabla} \tilde{\nabla} \tilde{U}$ where we need only $(\tilde{V} \tilde{V} \tilde{U})_{H H, H \cdots H}$ and $(\tilde{V} \tilde{V} \tilde{U})_{V V, H \cdots H}$. As the required calculation is straightforward we give here only the result,

$$
\begin{aligned}
& \left(\left(\tilde{\nabla} \tilde{\nabla} U^{L}\right)_{H H, H \cdots H}\right)_{k \jmath, \imath_{1} \cdots \imath}=\nabla_{k} \nabla_{j} U_{\imath_{1} \cdots \imath} p-\frac{1}{4} \sum_{a=1}^{p} R_{k j}{ }^{\tau} R_{\imath_{a}}{ }^{t}{ }^{\tau} U_{\imath_{1} \cdots t \cdots \imath^{\prime} p} \\
& -\frac{1}{4} \sum_{a=1}^{p} R_{k \imath_{a}}{ }^{\tau} R_{\jmath}{ }^{t}{ }_{\tau} U_{\imath_{1} \cdots t \cdots \tau_{p}},
\end{aligned}
$$

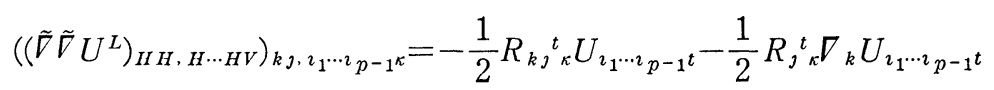

$$
\begin{aligned}
& -\frac{1}{2} R_{k}{ }_{\kappa} \nabla_{j} U_{i_{1} \cdots i_{p-1} t} \text {, }
\end{aligned}
$$




$$
\begin{aligned}
& \left(\left(\tilde{\nabla} \tilde{\nabla} U^{L}\right)_{H H, H \cdots H V V}\right)_{k_{j}, \imath_{1} \cdots \imath_{p-2} \kappa_{1} \kappa_{2}} \\
& =\frac{1}{4}\left(R_{k}{ }^{t}{ }_{\kappa_{1}} R_{j}{ }_{j \kappa_{2}}-R_{k}{ }^{t}{ }_{\kappa_{2}} R_{j \kappa_{1}}^{s}\right) U_{u_{1} \cdots p_{p-2} t s}, \\
& \left(\left(\tilde{\nabla} \tilde{\nabla} U^{L}\right)_{V V, H \cdots H}\right)_{\nu \mu, \imath_{1} \cdots \imath_{p}} \\
& =-\frac{1}{2} \sum_{a=1}^{p}\left(R_{\nu 2}{ }^{t}{ }_{\mu}+\frac{1}{2} R^{t s}{ }_{\nu} R_{\imath_{a} s \mu}\right) U_{\imath_{1} \cdots t \cdots \imath} \\
& +\frac{1}{4} \sum_{1 \leqq a<b \leqq p}\left(R_{\imath_{a}}{ }^{t}{ }_{\nu} R_{\imath_{b}{ }^{s}{ }^{s}}+R_{\imath_{a}}{ }^{t}{ }^{\prime \prime} R_{\imath_{b}}{ }^{s}{ }_{\nu}\right) U_{\imath_{1} \cdots t \cdots s \cdots v_{p} p}, \\
& \left(\tilde{\nabla} \tilde{\nabla} U^{L}\right)_{V V, H \cdots H V}=0, \quad\left(\tilde{\nabla} \tilde{\nabla} U^{L}\right)_{V V, H \cdots H V V}=0 .
\end{aligned}
$$

Let us define $\tilde{S}$ by

$$
\tilde{\nabla}_{T} \tilde{\nabla}^{T} \tilde{U}_{A_{1} \cdots A}=\tilde{S}_{A_{1} \cdots A p}
$$

where $\tilde{U}=U^{L}$. Then we get from the foregoing result

$$
\begin{aligned}
& \left(\widetilde{S}_{H \cdots H}\right)_{i_{1} \cdots \imath}=g^{k \jmath}\left(\left(\tilde{\nabla} \tilde{\nabla} U^{L}\right)_{H H, H \cdots H}\right)_{k_{\jmath}, \imath_{1} \cdots \imath p} \\
& \left.+g^{\nu \mu}\left(\tilde{\nabla} \tilde{\nabla} U^{L}\right)_{V V, H \cdots H}\right)_{\nu \mu, \imath_{1} \cdots \imath_{p}} \\
& =\nabla_{t} \nabla^{t} U_{\imath_{1} \cdots \imath_{p}}-\frac{1}{2} \sum_{a=1}^{p} R_{\imath_{a} s}{ }^{\tau} R_{\tau}^{t s} U_{\imath_{1} \cdots t \cdots \imath} \\
& +\frac{1}{2} \sum_{1 \leqq a<b \leqq p} R_{\imath}{ }^{t \tau} R_{\imath_{b}}{ }^{s} U_{\imath_{1} \cdots t \cdots s \cdots \imath}, \\
& \left(\widetilde{S}_{H \cdots H V}\right)_{i_{1} \cdots \imath_{p-1} \kappa}=-\frac{1}{2} g^{k j} R_{k j}{ }_{k} U_{\imath_{1} \cdots 2 p-1}-R^{t s}{ }_{k} \nabla_{t} U_{\imath_{1} \cdots \imath_{p-1} s}, \\
& \left(\tilde{S}_{H \cdots H V V}\right)_{i_{1} \cdots i p-2{ }^{\kappa_{1} \kappa_{2}}}=\frac{1}{2} R^{k t}{ }_{\kappa_{1}} R_{k}{ }^{s}{ }_{\kappa_{2}} U_{\imath_{1} \cdots p_{p-2}} t s .
\end{aligned}
$$

Substituting (2.6), (2.7), (2.8), (2.9), (2.10), (3.1), (3.3), (3.4), (3.5) and (3.6) into (3.2) we get

$$
\begin{aligned}
& \left(\left(\tilde{\Delta} U^{L}\right)_{H \cdots H}\right)_{i_{1} \cdots \imath_{p} p}=(\Delta U)_{i_{1} \cdots \imath_{p}}+\frac{1}{2} \sum_{1 \leqq a<b \leqq p} R_{\imath_{a} \imath_{b}}{ }^{\tau} R^{t s} U_{\tau} U_{i_{1} \cdots t \cdots s \cdots v_{p} p}, \\
& \left(\left(\tilde{\Delta} U^{L}\right)_{H \cdots H V}\right)_{i_{1} \cdots p_{p-1} \kappa}=R^{t s}{ }_{\kappa} \nabla_{t} U_{\imath_{1} \cdots i_{p-1} s}+\frac{1}{2} \sum_{a=1}^{p-1} R_{\imath_{a}}{ }^{t s} U_{\imath_{1} \cdots t \cdots p^{\prime} p 1^{s}}, \\
& \left(\left(\tilde{\Delta} U^{L}\right)_{H \cdots H V V}\right)_{i_{1} \cdots p_{p-2} \kappa_{1} \kappa_{2}}=\left(R_{\kappa_{1}}{ }^{t s}{ }_{\kappa_{2}}-R^{k t}{ }_{{ }_{1}} R_{k}{ }_{k}^{s}{ }_{\kappa_{2}}\right) U_{\imath_{1} \cdots v_{p-2} t s} .
\end{aligned}
$$

If $p=0$ we have only $\tilde{\Delta}_{\varphi}{ }^{L}=\Delta \varphi$. If $p=1$ we have only

$$
\left(\left(\tilde{\Delta} U^{L}\right)_{H}\right)_{i}=(\nabla U)_{i}, \quad\left(\left(\tilde{\Delta} U^{L}\right)_{V}\right)_{\kappa}=R^{t s}{ }_{\kappa} \nabla_{t} U_{s} .
$$


$\S 4$. Eigenelement $\omega$ of the Laplace-Beltrami operator $\Delta$ in $\left(B,{ }^{B} g\right)$ such that the lift $\omega^{L}$ is also an eigenelement of $\tilde{\Delta}$ in $(\tilde{M}, \tilde{g})$.

From (3.7), (3.8) and (3.9) we get the following Main Theorem.

THEOREM 4.1. Let $\omega=U_{i_{1} \cdots \imath_{p}} d x^{i_{1}} \wedge \cdots \wedge d x^{\imath_{p}}$ be an ergenelement of the LaplaceBeltram operator $\Delta$ in the base manifold $\left(B,{ }^{B} g\right)$ with eigenvalue $\lambda$. A necessary and sufficient condition that $\tilde{\omega}=\omega^{L}$ be an eigenelement of the Laplace-Beltram operator $\tilde{\Delta}$ in the total manifold $(\tilde{M}, \tilde{g})$ with the same eigenvalue $\lambda$ is that $\omega$ satisfy the following equations,

$$
\begin{aligned}
& \sum_{1 \leqq a<b \leqq p} R_{\imath_{a} \imath_{b}{ }^{\tau}} R^{t s}{ }_{\tau} U_{\imath_{1} \cdots t \cdots s \cdots \imath}=0, \\
& R^{t s}{ }_{k} \nabla_{t} U_{\imath_{1} \cdots \imath_{p-1} s}+\frac{1}{2} \sum_{a=1}^{p-1} R_{\imath_{a}}{ }^{t s}{ }_{\kappa} U_{\imath_{1} \cdots \imath \cdots \imath_{p-1} s}=0, \\
& \left(R_{\kappa_{1}}{ }^{t s}{ }_{\kappa_{2}}-R^{k t}{ }_{\kappa_{1}} R_{k}{ }_{\kappa_{2}}^{s}\right) U_{\imath_{1} \cdots 2 p-2} t s=0 .
\end{aligned}
$$

From this theorem we get a simpler theorem,

THEOREM 4.2. Let $\omega$ be an eigenelement of the Laplace-Beltrami operator $\Delta$ in $\left(B,{ }^{B} g\right)$. A sufficient condition that $\tilde{\omega}=\omega^{L}$ be an eigenelement of $\tilde{\Delta}$ in $(\tilde{M}, \tilde{g})$ is that $\omega$ satisfy the equations

$$
\begin{aligned}
& R^{t s}{ }_{\kappa} U_{\imath_{1} \cdots \imath p-2} t s=0, \\
& R^{t s}{ }_{\kappa} V_{\imath_{1} \cdots \imath_{p-1} t s}=0
\end{aligned}
$$

where $d \omega=V_{\imath_{1} \cdots \imath_{p+1}} d x^{\imath_{1}} \wedge \cdots \wedge d x^{2 p+1}$.

Proof. $(\alpha)$ is satisfied by $(\delta)$. From $(\delta)$ we get

hence

$$
R_{\imath_{1}}{ }^{t s \kappa} U_{\imath_{2} \cdots \imath p-1} t s+R^{t s \kappa} \nabla_{\imath_{1}} U_{\imath_{2} \cdots \imath p-1}{ }=0
$$

$$
\sum_{a=1}^{p-1} R_{\imath_{a}}{ }^{t s \kappa} U_{\imath_{1} \cdots t \cdots \imath^{\prime} p-1^{s}}+R^{t s \kappa} \sum_{a=1}^{p-1} \nabla_{\imath_{a}} U_{\imath_{1} \cdots t \cdots \imath_{p-1}}=0 .
$$

From $(\varepsilon)$ we get

$$
R^{t s \kappa} \sum_{a=1}^{p-1} \nabla_{\imath_{a}} U_{\imath_{1} \cdots t \cdots \imath_{p-1} s}-2 R^{t s \kappa} \nabla_{t} U_{\imath_{1} \cdots \imath p-1^{s}}=0 .
$$

This proves that $(\beta)$ is satisfied. From $(\delta)$ we also get

$$
\left(\partial_{\kappa_{1}} R_{{ }_{\kappa_{2}}}^{t s}-\left\{\begin{array}{c}
\lambda \\
\kappa_{1} \kappa_{2}
\end{array}\right\} R_{\lambda}^{t s}\right) U_{\imath_{1 \cdots i} p-2 t s}=0
$$

which proves that $(\gamma)$ is satisfied in view of (2.3). 
Concerning $(\delta)$ and $(\varepsilon)$ we get the following theorem.

THEOREM 4.3. Let $\omega$ be a p-form salusfying $(\delta)_{p}$ and $(\varepsilon)_{p}$. Then $d \omega$ and $\delta \omega$ satisfy $(\delta)_{p+1},(\varepsilon)_{p+1}$ and $(\delta)_{p-1},(\varepsilon)_{p-1}$ respectively.

Proof. That $\omega$ satisfies $(\varepsilon)_{p}$ is equivalent to that $d \omega$ satisfies $(\delta)_{p+1}$. Moreover $d \omega$ always satisfies $(\varepsilon)_{p+1}$ in view of $d d=0$. Thus Theorem 4.3 is proved for $d \omega$. From

$$
R^{t s \kappa} U_{j i_{1} \cdots p_{p-3} t s}=0
$$

we get

$$
R^{j t s \kappa} U_{j i_{1} \cdots{ }^{2} p-3} t s+R^{t s \kappa} \nabla^{J} U_{j i_{1} \cdots 2 p-3} t s=0 .
$$

As $R_{k j i}{ }^{\kappa}$ satisfies $(2.4)$ we get

$$
R^{t s \kappa} \nabla^{\jmath} U_{j i_{1} \cdots 2 p-3 t s}=0
$$

which proves that $\delta \omega$ satisfies $(\delta)_{p-1}$. As $\omega$ is an eigenelement of $\Delta$ we have $d \delta \omega=\lambda \omega-\delta d \omega$. As $d \omega$ satisfies $(\delta)_{p+1}, \delta d \omega$ satisfies $(\delta)_{p}$. Hence $d \delta \omega$ satisfies $(\delta)_{p}$. This proves that $\delta \omega$ satisfies $(\varepsilon)_{p-1}$ and completes the proof.

Applying the result obtained above to harmonic forms we get

THEOREM 4.4. Let $\varphi=U_{i_{1} \cdots \imath_{p}} d x^{\imath_{1}} \wedge \cdots \wedge d x^{2} p(p \geqq 2)$ be a harmonic form of $\left(B,{ }^{B} g\right)$. Then $\varphi^{L}$ is a harmonic form of $(\tilde{M}, \tilde{g})$ if $\varphi$ satisfies $(\delta)$. The lift of any harmonic 1-form of $\left(B,{ }^{B} g\right)$ is a harmonic 1 -form of $(\tilde{M}, \tilde{g})$.

Let $(\tilde{M}, \tilde{g}, \tilde{\xi})$ be a Sasakian manifold [9]. Let there be a Riemannian submersion $\pi:(\tilde{M}, \tilde{g}) \rightarrow(M, g)$ called a Sasakian submersion [6], [10], [11]. In this submersion fibers $F$ are generated by the Killing vector field $\tilde{\xi}$. As $\operatorname{dim} F=1$ we can write $R_{j i}$ instead of $R_{j i}{ }^{k}$. $\frac{1}{2} R_{\jmath}{ }^{2}=F_{j}{ }^{2}$ represents a complex structure $J$ such that $(J, g)$ is a Kähler structure on $M$. Hence $R_{j i}$ is a harmonic 2-form. This does not satisfy $(\alpha)$ since $(\alpha)$ assumes the form

$(\alpha)_{2}$

$$
R_{j i} R^{t s} U_{t s}=0
$$

if $m=1, p=2$. This gives an example of harmonic forms of $\left(B,{ }^{B} g\right)$ whose lift is not a harmonic form.

\section{REFERENCES}

[1] M. Berger, P. Gauduchon, E. Mazet, Le spectre d'une variété Riemannienne, Springer-Verlag 1971.

[2] R.H. Escobales, Riemannian submersions with totally geodesic fibers, J. Differential Geometry, 10 (1975), 253-276.

[3] S. Ishihara, Vector fields in fibred spaces with invariant Riemannian metric, Differential Geometry, in honor of K. Yano, Kinokuniya, Tokyo, 1972, 163-178. 
[4] S. Ishihara And M. Konishi, Differential geometry of fibred spaces, Publications of the study group of geometry, Tokyo, Vol. 8 (1973).

[5] Y. Mutō, On some properties of a fibred Riemannian manifold, Sci. Rep. Yokohama Nat. Univ., Sect. I, No. 1 (1952), 1-14.

[6] Y. Mutō, Riemannian submersions and critical Riemannian metrics, J. Math. Soc. Japan, 29 (1977), 493-511.

[7] T. Nagano, On fibred Riemannian manifolds, Sci. Papers College Gen. Ed. Univ. Tokyo, 10 (1960), 17-27.

[8] B. O'Neill, The fundamental equations of a submersion, Michigan Math. J., 13 (1966), 459-469.

[9] S. Sasaki and Y. Hatakeyama, On differentiable manifolds with contact metric structures, J. Math. Soc. Japan, 14 (1962), 249-271.

[10] K. Yano And S. Ishihara, Differential geometry of fibred spaces, Kōda1 Math. Sem. Rep., 19 (1967), 257-288.

[11] K. YANO AND S. IshIHARA, Fibred spaces with invariant Riemannian metric, Kōdai Math. Sem. Rep., 19 (1967), 317-360.

2262-150, TOMIOKA-CHO, KanAZAWA-KU, YOKOHAMA 236, JAPAN 